Article

\title{
Design of Digital Interaction for Complex Museum Collections
}

\author{
Laura Loredana Micoli $(\mathbb{D}$, Giandomenico Caruso $(\mathbb{D}$ and Gabriele Guidi * (D) \\ Department of Mechanical Engineering, Politecnico di Milano, Via Giuseppe La Masa 1, 20156 Milan, Italy; \\ laura.micoli@polimi.it (L.L.M.); giandomenico.caruso@polimi.it (G.C.) \\ * Correspondence: gabriele.guidi@polimi.it
}

Received: 10 May 2020; Accepted: 17 June 2020; Published: 22 June 2020

\begin{abstract}
Interactive multimedia applications in museums generally aim at integrating into the exhibition complementary information delivered through engaging narratives. This article discusses a possible approach for effectively designing an interactive app for museum collections whose physical pieces are mutually related by multiple and articulated logical interconnections referring to elements of immaterial cultural heritage that would not be easy to bring to the public with traditional means. As proof of this concept, a specific case related to ancient Egyptian civilization has been developed. A collection of Egyptian artifacts such as mummies, coffins, and amulets, associated with symbols, divinities, and magic spells through the structured funerary ritual typical of that civilization, has been explained through a virtual application based on the concepts discussed in the methodological section.
\end{abstract}

Keywords: cultural heritage exhibition; digital heritage; intangible heritage; virtual museum; multimedia; ancient Egypt; interactive devices

\section{Introduction}

The international spectrum of museum institutions is highly diversified in terms of size of the structures, location and geographical accessibility, type of cultural proposal, history of the institutions and economic availability. The 26th edition of Museums of the World [1] counts more than 55,000 museums in 202 countries, ranging from large historical institutions with collections of international appeal to small corporate or ethnographic museums in the provinces.

According to the International COuncil of Museums (ICOM) most recent definition, the role of museums is to "hold artefacts and specimens in trust for society, safeguard diverse memories for future generations" and "collect, preserve, research, interpret, exhibit, and enhance understandings of the world" [2].

In other words, the "understanding" function is a crucial purpose of museums since the exhibited objects frequently hide meanings not made explicit by their physical appearance alone. Therefore, every museum piece implicitly possesses the possibility to narrate different stories that may reveal the object's genesis, use, cultural context, history, legends and more. Knowing these can make it clearer to the visitor why that object is important, how it relates to a broader context, and why it is worthy of preservation and display in the first place.

Providing these immaterial pieces of information that clarify the role and function of a single object becomes even more important when connecting a specific cultural heritage piece with others exhibited in the same collection in a museum, especially when this collection is on display, because it is a coherent body of artifacts linked together by a common historical, motivational, religious or conceptual background. 
This last point will not be news to curators, but using the traditional tools for museum display, such as panels and labels placed in the various vitrines, can require a lot of physical space, significant financial resources, and may not be very effective in terms of promoting understanding.

Digital approaches and tools, in their various forms, are now a valid support for museum installations, especially when such linking pieces of information is one of the primary interpretive aims of an exhibition. Digital solutions can guide visitors within articulated collections through narrative paths that make more evident the logical connections between different elements, which may not be not explicit.

Furthermore, using 3D digital reproductions of the objects can give rise to exhibits of various kinds by relating multiple museum pieces through shared immaterial elements that can be pointed out and narrated in a virtual environment. Ancient everyday objects, whose shapes would normally be incomprehensible to a museum visitor, can be inserted into an explanatory virtual context to reveal their practical function, for example through a 3D animation. Similarly, ancient ritual objects, whose rationale is inexplicable when examined out of context, can be effectively explained through a narration of the ritual or by simulating the objects' symbolic role with multimedia support and digital enhancements.

Crucial components of the physical objects preserved in the museums include all the immaterial elements connected to them. They become useful links for any form of narration implemented around an exhibited piece, in order to achieve an enhanced understanding of the whole collection.

Here we report on an approach that we pursued to achieve this result through the convergence of different narrative modalities and media that Henry Jenkins defined as "Transmedia storytelling" [3]. This is a narrative form that, moving through different types of media, contributes to completing and integrating the user experience with complementary information. Each medium, conveying new and distinct pieces of information, contributes to the development of the history and the understanding of the world whose story is being narrated. In this way, the user finds it possible to reconstruct the overall meaning of a cultural asset by mentally integrating various media.

This narrative potential has always been available to conventional exhibition designers [4], but it finds a new and more extensive application in museums making use of digital technologies for standard practices such as classification, archiving, collection and exhibition [5]. These basic digital practices make possible "museum object lessons" for explaining historical concepts though digitized objects [6].

\subsection{Digital Technologies for Museum Exhibits}

The technological approaches developed for interacting with items that are instantiated either in the real world as actual museum objects or in the virtual world through their digital simulacra are generally classified in terms of their position on the so-called reality-virtuality continuum. This concept dates back 25 years when Milgram et al. first introduced Augmented Reality (AR), juxtaposing it to Virtual Reality (VR) [7]. All these digital technologies, collectively known as Mixed Reality (MR), allow us to navigate from the virtual world to the real one with different degrees of immersivity in the interplay between real and digital objects. MR possesses great potential for cultural learning $[8,9]$.

Applications of MR in museums have exploited web-based approaches for remotely interacting with a museum exhibition through a desktop computer, sometimes involving the use of a web-based virtual museum using 3D game engines like Unity 3D, Unreal or CryEngine to support rich content in distributed Web resources [10,11]. Such applications, if designed for accessing the museum from home, can also effectively deliver virtual visits to the museum itself in the form of virtual kiosks [12,13], which are useful for combining a real visit with complementary information.

Regarding the implementation at the museum, in recent years all types of immersive technologies have been used, ranging from special projection rooms, similar to small 3D cinemas for showing stereoscopic images to a public equipped with active or passive stereoscopic glasses [14], to wearable stereoscopic devices for gaming like the Oculus Quest or HCT Vive, made available at the museum site [15], sometimes enhanced by the ability to detect the user device's position [16]. All these 
approaches, although highly engaging, require specific spaces as well as safety measures for protecting the visitors as they move about without seeing the reality around them. Indeed, accidents such as falls or collisions with other users or objects are possible when such devices are used. The safety measures add significant costs and liability militating against their use by museums.

The practical usability of AR in a public space as well the possibility of using the real world as an informative background-intrinsically 3D by nature-has made AR very successful in museums [17] in a variety of ways. The simplest approach, based on a monoscopic augmentation of the real scene, makes use of personal devices like smartphones or tablets owned by the visitors. In this case, the most critical issue of AR is the connection of the augmented content with the real scene. This can be done by using fiducial markers [18], featured images of the scene, or the 3D content of the scene itself. The latter approach is particularly effective but requires a knowledge of the 3D shape of the environment by the mobile device used for delivering AR. In this way, by matching the 3D scene seen by the device with a 3D scene previously known by the app, the six degrees of freedom (6DOF) identifying the position in space of the device can be estimated and the augmented content appropriately rendered over the virtual scene. Today, this feature is starting to be available on tablets [19] and smartphones now [20], but this is a recent development. For this reason, museum applications involving precise scene augmentation have previously required tablets equipped with special reflective 3D targets monitored by cameras distributed in the environment for tracking the 6DOF of each device. The result is undoubtedly spectacular [21], but, again, it also requires a great financial investment, often not possible within standard museum budgets.

The latest specialized AR devices such as the Microsoft Hololens or the widely anticipated but as-yet unreleased Apple Glass employ a similar real-time 3D scanning technology for improving the estimation of the device's 6DOF with the addition of a stereoscopic visualization and AR processing made directly onboard (Hololens), or in a small processing unit like a smartphone connected wirelessly (Apple Glass). Once again, the cost of each device and the personnel cost for sanitizing, distributing and controlling its circulation among the visitors would be beyond the budget of an average museum.

\subsection{Design Strategies for Digital Museums}

Despite the cost, it is evident that many useful technological building blocks are now available for creating digital applications that digitally enhance museum visits. However, in addition to technology, the design framework for making such technologies effective is also critically important. International research efforts to define proper criteria for developing digital interactions in museums have been made since the 1990s [22-24]. Nevertheless, in recent years, the main boost has arrived from the European Commission, which in the period 2014-2018 invested $€ 366 \mathrm{M}$ in the museum domain through various funding programs: Creative Europe: Culture; Creative Europe: Media; Erasmus Plus; Europe for Citizens; Horizon 2020 (social sciences and humanities). As a result, 633 different projects have been developed, involving 942 partners from 521 European organizations [25]. Some of these projects have made important contributions to this domain, defining general design frameworks for developing interactive narrations in a museum environment. A few of these may be cited:

- $\quad$ ARCO (Augmented Representation of Cultural Objects) for the first time provided a complete solution for the digitization, management and the presentation of virtual museum exhibitions, addressing visualization in interactive VR and AR interfaces by adopting a component-based approach and supporting the mixing and matching of individual components through the use of the Extensible Markup Language (XML) for interoperability purposes [26];

- $\quad$ CHESS (Cultural Heritage Experiences through Socio-personal interactions and Storytelling) researched, implemented and evaluated an innovative conceptual and technological framework to integrate interdisciplinary research in personalization and adaptivity, digital storytelling, interaction methodologies, and narrative-oriented mobile and mixed-reality technologies [27]. The current interactive visit at the Athens acropolis, for example, is based on this system; 
- EMOTIVE was the evolution of CHESS, based on the premise that cultural sites are highly emotional places. In the framework of this project, the consortium has researched, designed, developed and evaluated methods and tools that can support cultural and creative industries in creating narratives and experiences focused on the emotional storytelling of cultural objects [28];

- MESCH (Material EncounterS with digital Cultural Heritage) investigated the design and use of tangible smart replicas to interact with digital content within a museum exhibition. These smart replicas are used to access additional layers of content that complement the traditional information provided about objects in a museum [29].

In contrast with the research just mentioned, the research we present here was focused on a specific aspect: how to exploit MR interaction to support a museum visit when a large intangible heritage component represents the connecting element between the objects of an exhibition. Hence, the purpose of this paper is to show a possible design approach for developing a low-cost digital interactive application aimed at explaining a type of collection identified as "complex" according to the definition given in Section 2. Our approach is to use as linking components the immaterial elements that are "hidden" within each exhibited physical object and which are particularly rich in complex collections. We use as an example a "complex collection" of a specific set of ancient Egyptian artifacts linked together through the articulated funerary ritual typical of that civilization. Owing to the "low-cost" feature and the solution's expected use in a generally crowded museum, the implementation is based on an interactive information kiosk making use of 3D models and other media rather than immersive technologies. Our focus is on the development process involving an interdisciplinary team and the consequent organization of the information/interactions provided by our application to make "readable" exhibited objects whose meaning is otherwise obscure to the visitor.

\section{Methodology}

\subsection{Complex Museum Collections}

The various forms of non-physical heritage gained more importance after UNESCO issued a convention for safeguarding of "intangible cultural heritage" (ICH) in 2003 [30]. In that convention, for the first time ICH was defined as a domain including: a) oral traditions and expressions, including language as a vehicle of the intangible cultural heritage; b) performing arts; $c$ ) social practices, rituals and festive events; d) knowledge and practices concerning nature and the universe; e) traditional craftsmanship.

The literature on this topic has pointed out that "tangible heritage, without intangible heritage, is a mere husk or inert matter" [31]. This has recently led to research stressing the importance of avoiding the dichotomy between tangible and intangible heritage as initially stated, considering any cultural object as a multifaced entity incorporating both tangible and intangible elements that deserve to be included in its description through a specific semantics [32]. This undoubtedly should have an impact on the criteria for creating a museum collection.

The term "collection" has historically involved several meanings and nuances associated with different levels of connection among the items that a specific grouping of objects includes. Starting from the Renaissance, when a learned elite developed a new passion for collecting valuable items to be exhibited for the purpose of gaining social recognition and increasing personal prestige, the criteria for defining how to group objects used to form a collection have significantly evolved. In the early days, the choice was mainly driven by notions of meaningful proximity, juxtaposition, or alignment, indicating underlying symbolic resemblance [33]. Later, during the seventeenth century, new ideas about how to organize and order objects into meaningful collections began to supersede some of those that had informed earlier practices. In particular, the idea that there were multiple forms of resemblances, connected by complex and cryptic linkages, came to be replaced mostly by the concept that physical similarities or dissimilarities between things could themselves point directly to the natural scheme. Such an idea led to the development of strongly taxonomic criteria that were first introduced in science and consequently in the requirements for creating museum collections [34]. In the 
nineteenth century, the criteria have further evolved, leading to various plausible classification grids lying in several disciplines such as anthropology, ethnology, archaeology, natural history, and science, supported by chronological and geographical differences [35]. With the twentieth century, questions about the legitimacy of existing classificatory categories for creating collections were increasingly raised in museums. The evolutionary process of the cultural classification of collectible items has therefore led to a new level of flexibility in contemporary museology regarding the criteria underlying the creation of a group of objects signified by the word "collection" [36].

As a result, now we may have many different levels of semantic connection among the items belonging to a collection, also involving their immaterial components. For this reason, we introduce here the concept of "collection complexity," associating it to the density of such connections. Such a density is a parameter motivating the development of a digital mediator for explaining a collection to the general public. The less obviously the physical objects themselves express such semantic connections, the more helpful the contribution of the digital mediator can be for explaining to the public the role and purpose of any specific object exhibited in a collection.

In particular, we can define three different levels of complexity:

- Low - the group is formed by entirely unrelated objects, whose grouping is justified by the mere function or symbolism that they represent rather than each object's mutual relationship with the others. An example of this kind is the collection of the "Museum der Dinge" (Museum of Things) in Berlin (https://www.museumderdinge.org). The readability of the collection is implicit in the objects themselves;

- Medium - the grouped selection of objects follows the general criteria, such as belonging to the same civilization, historical period, artistic or cultural movement, that makes it somehow homogeneous. In this case, a moderate number of semantic interconnections among the members could be present (e.g., paintings created by the same artist, different sculptural representations of the same divinity, etc.). Some complementary explanations should help the readability of this level of complexity;

- High - the group is made of objects strictly interlinked by many semantic connections associated to ICH components that are possibly non-explicit, such as being associated with the same person for specific activities or being used for rituals that connect each one to the others.

In the latter case, many complementary explanations are needed to make clear the reason for assigning each object to the collection. Such extensive descriptions are due both to the mutual relationships among the items in the collection as well as the connection linking them, represented by an immaterial element such as a ritual. In this paper, we refer to the latter case as a "complex museum collection" (CMC). A collection of objects associated with the funerary rituals of ancient Egypt is a typical example of a CMC.

\subsection{Digital Interaction with $C M C s$}

CMCs could benefit more than other kinds of collections from the use of technological aids to reveal the complexity embedded in its objects. Such aids might include virtual models, multimedia supports, and hyperlinks for delivering specific pieces of information not explicitly deductible by the objects alone. Moreover, if we wish to do something more than merely explain the function of each item in a collection, this solution is particularly suitable because it works well with the narrative tools that can more effectively make clear to visitors the articulated connections among the items belonging to a CMC.

In the work of Katifori et al. [37], the best practices for designing interactive storytelling for a museum application are well summarized. These practices can be usefully reapplied when designing other digital interactive applications whose development involves expertise in a wide array of subjects. 
In order to converge on a feasible interactive application, the experts participating in the design process need to have a shared vision about the meaning of the interactive narrative and focus on it, while at the same time, keeping in mind that stories refer to people and not just to exhibited objects.

If the interactive application is created to be used inside a museum, the physical space must be accurately designed, and it is necessary to aim at the right balance between the digital and the real context. For example, if a digital installation is used, it is essential to ensure that it does not absorb all the user's attention, as the digital narration is conceived as a support and not as an alternative to the experience of the real museum.

In particular, [37] suggests the need for a multidisciplinary team to develop an authoring workflow devoted to interactive storytelling in a cultural heritage context. Starting from this suggestion, we derived a definition of roles suitable to develop a digital application aimed at better understanding a CMC:

- Domain experts-these include museum curators and experts in museum content and topics (archaeologists, historians, art history experts, etc.). They provide knowledge about the available assets and possible interpretative keys useful to define the application content;

- Exhibition designers-they are responsible for the set-up of the museum exhibitions, including both the physical cultural heritage assets and also the devices running the digital applications;

- Content designers-they provide the connection framework of the material and immaterial cultural heritage assets; they define the possible paths of the interaction and create the textual contents;

- Digital asset designers-they oversee the digital asset creation and the combination of such assets into interactive activities.

The primary team's goal is to generate a synthesis of all the elements able to disclose the multiple connections between the physical asset of the CMC through their immaterial components.

The design phase starts by discussing the contents with the domain experts, discovering the consistency of the collection, and highlighting the assets' meanings and the possible interpretative issues. This step of the work is critical, because everything is put on the table as raw material, with all the connections highlighted, leaving open any possible narrative solution. In the case of CMCs, this step might be somewhat challenging for the number of elements and meanings involved, which can generate a dense graph of connected nodes, representing physical objects, intangible objects, and connecting branches, such as the one shown in Figure 4.

The subsequent discussion should lead to defining (i) the more representative physical items of the collection to be inserted in the application, and (ii) the framework for developing the application and the proposed interaction levels.

These steps could be iterated to refine the result, progressively integrating as many suggestions as possible provided by the different domain experts and the discussions among them.

Once the multidisciplinary team defines the key concepts to be communicated by the digital application, it is useful to consider the digital items that can be used to connect to the material or immaterial cultural heritage items to be explained by posing crucial questions such as: (i) "what are the digital items more suitable to enhance the understanding of a specific CMC among texts, metadata, images, drawings, videos, 3D models, sounds?" (ii) "What are the digital items already existing and exploitable?" (iii) "What type of digital items need to be generated since they are not already available?"

The ideal implementation usually must deal with the real conditions of the exhibition area and the available economic resources. Often museums are in pre-existing venues such as ancient buildings, often not conceived for accommodating an exhibition.

The real available exhibition space is an aspect that significantly impacts the design of an interactive application for a specific CMC in a particular museum. In the case of interactive installation, it is necessary to consider, for example, the size of the space available to install the equipment for the digital application. The required area can range from tens of square meters for a VR cave, a square meter in the case of an interactive kiosk, or even less in the case of handheld devices. 
Furthermore, the maximum number of people who can access the digital application simultaneously has to be considered, in order to design a proper audience flow, choosing the best solution in terms of the duration of the interaction and the location of the kiosk in the exhibit area, so as to prevent the accumulation of visitors in line for accessing the interactive application close to the display vitrines showing the collection.

The acoustic constraints must also be considered during the planning phase, evaluating whether the museum can create isolated acoustic areas for making decisions about possible sounds and music provided by the application. If isolated areas are available, the sounds originated by the interactive installation can be distributed through loudspeakers. If not, headphones could be made available for the visitors, or alternatively, the application could be designed entirely soundless.

Summarizing the real space condition could lead to a series of limitations for:

- Tools that need ad hoc space for projections or the recording of user movements (e.g., virtual reality caves, boxes equipped with VR headsets, tracking sensor, etc.);

- Sound and audio effects that could be disturbing the rest of the exhibition;

- Use of the virtual application by individuals or small groups, for a limited time, for not creating gatherings that disturb the flow of other visitors.

Concerning the budgetary commitment, at least in Italy and other southern European countries, it frequently happens that state museums, despite the incredible richness and value of the collections owned, have minimal funds for managing exhibitions. Therefore, museums tend to avoid excessively expensive devices, due to the impact on the budget of both the initial purchase and their long-term management. The preferred technological devices for VR, such as wearable stereoscopic headsets, can become an impossible choice for different reasons.

Units with excellent performances are now affordably priced at just a few hundred euros. However, the arrangement of a set of devices, sufficient for covering hundreds of visitors that might be willing to experience a VR visit at once, can quickly multiply the cost to several thousands of euros, making it not compatible with the budget of most small and medium-sized museums.

Even if in relative terms, the value of a few hundred euros per each VR headset can be considered low in comparison with the amount of technology it includes, renting it to a museum visitor involves formal steps that dedicated personnel should carry out. For example, taking the ID of each visitor that borrows a VR unit, and giving it back when the visitor returns the headset.

Passing a wearable device from one visitor to another also poses issues in terms of the sanitization of each headset, which should be adequately disinfected after each use, engaging probably other dedicated personnel.

Experimental cases and literature show how, depending on the situation and the combination of the constraints, the highest communicative efficacy can be obtained with the latest generation of systems, which provide a high level of immersion, as well as with the use of less immersive systems, for example, touchscreen kiosks [13], more immediately accessible in terms of both implementation and user interaction.

\section{Case Study}

The implementation of the concepts illustrated above was addressed through the design of an interactive digital application related to the ancient Egyptian collection of the Milan Civic Archaeological Museum. The project, named PERVIVAL—Virtual Paths for the Improvement of Complex Museum Collections in the Milan Area (http://www.pervival.polimi.it/) — has been developed over a two-year timespan (2018-2020).

\subsection{The Specific CMC}

The collection is made up of a rather large number of items, such as the mummies of humans and animals, coffins and sarcophagi with hieroglyphic inscriptions, small statues, amulets, vases, etc. 
All these objects belong to different historical periods ranging from the so-called Middle Kingdom (2050-1710 BC) to the Ptolemaic Period (332-30 BC) of ancient Egypt. Therefore, as for the curation of a museum exhibition, the starting point for conceiving a digital application was to define a core narration around a specific theme, for connecting at least a few significant pieces of the collection. This choice was made together with the museum direction, supported by experts in Egyptology. They defined the body of funerary rituals related to the passage of the deceased from life to the Afterlife as the fil rouge to be made explicit for connecting the exhibited items each to other.

In ancient Egypt, there was a belief that the cosmos was divided into several parts: the earth corresponding to the world of the living, the world of the dead called Duat, and the Fields of Aaru (or Field of Reeds) was the heavenly paradise where the god Osiris rules and the deceased live forever. Each Egyptian aspired to eternal life, and to obtain it a multitude of actions and rituals were performed. The main action was to preserve and nurture the body and soul of the deceased to help them to overcome all the challenges they would have had to face in order to earn eternal life.

The remains that ancient Egyptian culture has left us are often part of such an elaborated set of rituals where each element has a specific meaning and is connected to the others by a network of semantic values [38-43]. This multiplicity of objects, rich in symbolism, can be considered paradigmatic of CMCs and represents an ideal context for studying and implementing digital solutions devoted to innovating and enhancing the educational mission of the museum.

In order to transfer these archaeological explanations into engaging narrative form, a subset of grave goods from the entire collection belonging to the museum was chosen and digitized. In particular:

- The mummy of Peftjauauiaset (hereafter "Pef" for short), his painted anthropoid bivalve coffin and the painted sarcophagus used for containing it (7th century BC). Pef was a scribe from Thebes, i.e., a person educated in the arts of writing, that was one of the most prominent figures in the royal court [44];

- The painted anthropoid bivalve coffin of Tesbastetperet (hereafter "Tes" for short), and the related wooden sarcophagus (7th-6th century BC). She was a wealthy woman part of the court: the coffin inscriptions describe Tes as "the lady of the house";

- Four canopic jars, used for storing the different internal organs of the deceased;

- Eighteen amulets of different types, including the important "hearth scarab," inscribed with microscopic spells, very difficult to be read under the naked eye when the object is in a showcase;

- A couple of ushabti, representing the hundreds that it is possible to find related to a deceased, and a painted box to store them;

- A false door stele, representing the passage from the Life to Afterlife world;

- A papyrus representing the "book of the dead", a collection of magic spells intended to assist the deceased during their journey to the Duat. This exemplar belonged to Hornefer, a priest and scribe lived in the Ptolemaic Period (305-30 BC).

\subsection{Digitization}

Except for the papyrus, the primary element to represent and narrate the objects were textured 3D models at high resolution that allowed the user to better analyze the artefacts, including those exhibited, making the information about them more accessible through an enriched navigation from one detail to another, supported at times by additional multimedia elements such as videos, images, drawings and texts.

Most of the 3D models were generated through digital photogrammetry, in different sessions from December 2016 to June 2017 (Figure 1).

The photo cameras employed were:

- $\quad$ An APS-C mirrorless camera Sony Alpha 6000 coupled with a Zeiss $24 \mathrm{~mm}$ f/1.8 lens;

- A full-frame Canon 5D Mark II digital reflex with two lenses: Canon EF 20mm f/2.8 USM and Canon EF $50 \mathrm{~mm} \mathrm{f/1.8.}$ 
For each camera-lens combination, the images were taken at a camera-object distance short enough to provide a ground sampling distance (GSD) not more than $0.25 \mathrm{~mm}$.

The SFM/IM (structure from motion/image matching) photogrammetric processing was carried out with the commercial package Agisoft Metashape. The reprojection error of the key points after the orientation phase was always below 0.5 pixel. The dense matching was performed systematically using $4 \times 4$ image sub-windows as the matching element, so that the final resolution of the $3 \mathrm{D}$ dense cloud was $1 \mathrm{~mm}$ or better.

The 3D model was generated from the meshing function included in the Metashape software package, using a high number of polygons $(2 \times$ the size of the dense cloud) in order to maintain the fine $3 \mathrm{D}$ details gathered through the photogrammetric campaign.

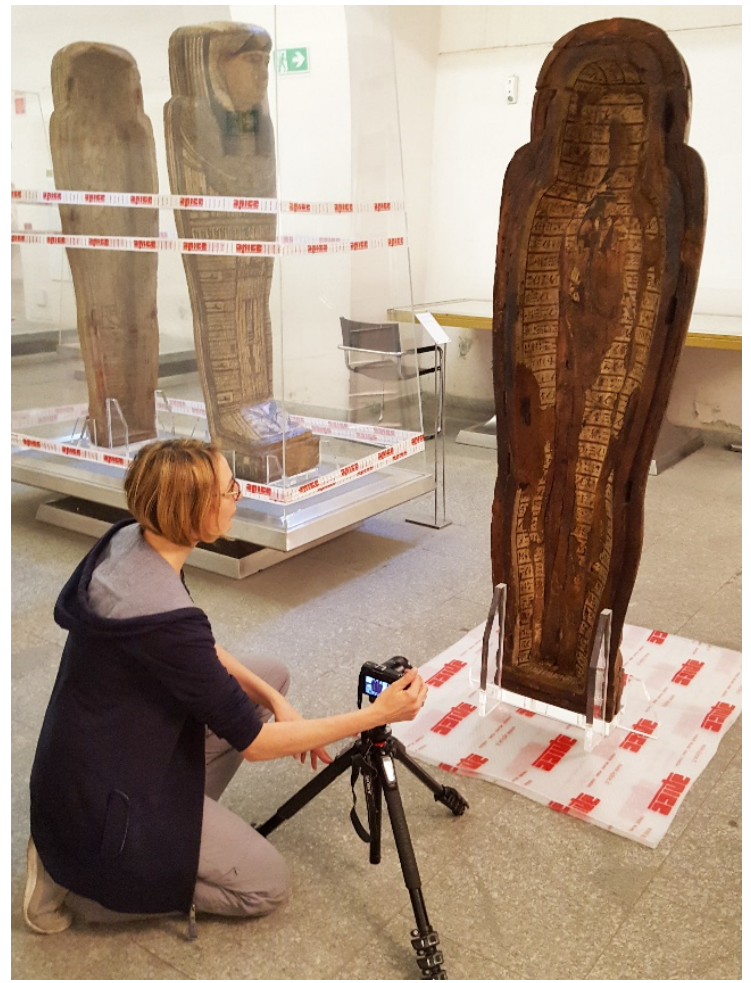

(a)

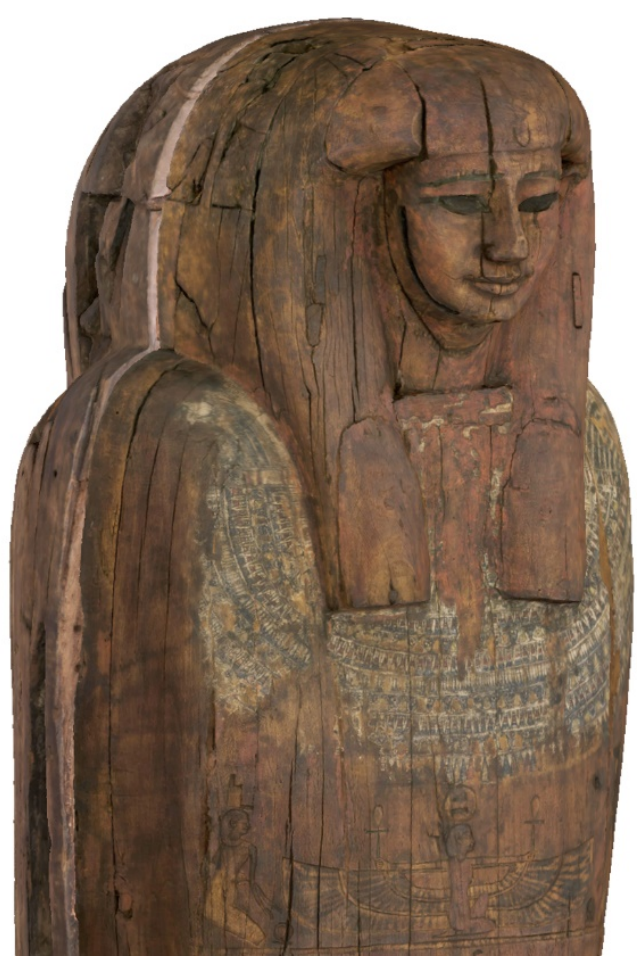

(b)

Figure 1. Anthropomorphic coffin of Peftjauauiaset: (a) photographic survey of the posterior valve for reconstruction using the SFM/IM (structure from motion/image matching) system, carried out at the Civic Archaeological Museum of Milan during the dismantling of the exhibition area for modernization works; (b) detail of the 3D model with a high-resolution texture of the two valves together.

The very large mesh, of course not suitable for a VR application owing to its size, passed a topology check/correction operated with the Open Source software package Meshlab [45], and was then simplified with a selective algorithm included in the software package Polyworks IMCompress, which is able to modulate the simplification level depending on the level of detail of each part of the model. A high-resolution retexturing in Metashape completed the modeling process.

It should be noted that part of the 3D models used in the museum application were reused from the European project 3DICONS, which took place between 2012 and 2015, using the same modeling protocol [46].

The purpose of 3DICONS was to publish the cultural heritage resources in the form of 3D models, making them accessible through the Europeana portal (www.europeana.eu). Such models have been here reused for two reasons. First, a significant starting dataset allowed the project to focus its resources on the development of the interaction model, and the experimental prototype in the museum, 
rather than on the creation of the digital content. Secondly, the fact that a good part of the material used in the museum application was also catalogued in Europeana created a useful connection to a potentially vast public represented by the end-users of the Europeana portal. The virtual application here described can be imagined as an articulated form of visualization and interaction that could be theoretically triggered by a standard Europeana search.

In addition to the photogrammetric survey techniques, nowadays, used in similar archaeological projects [47], some items have been analyzed using medical technologies as CT (computerized tomography), already presented in the literature to study the internal details of some archaeological findings with non-invasive techniques [48]. Such a study was conducted either on objects like the interiors of Pef's coffin, in order to explore their internal wooden structures and how the coffin was assembled (Figure 2), or Pef's mummy (Figure 9), which revealed interesting clues about the mummified body not otherwise attainable.

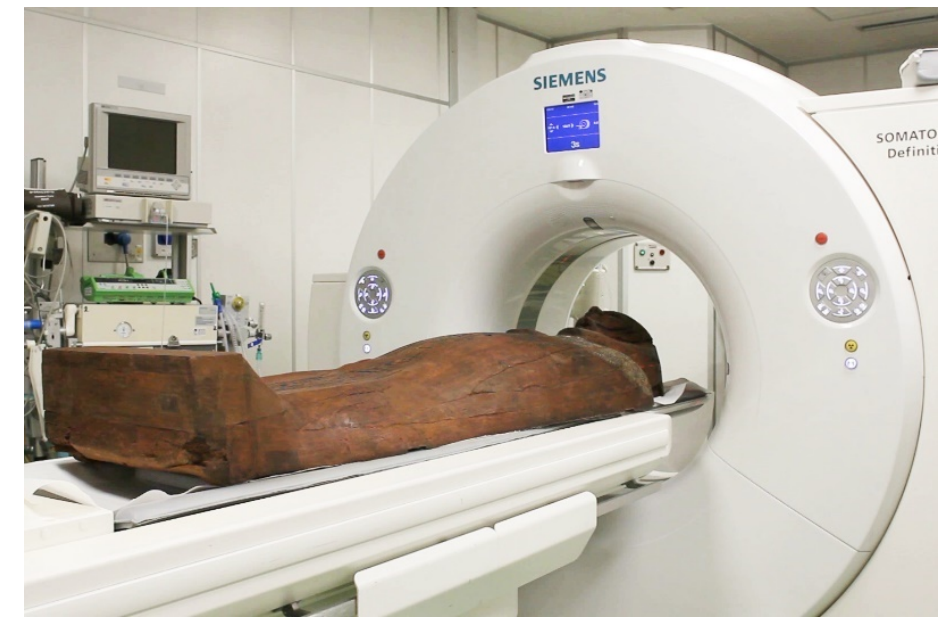

(a)

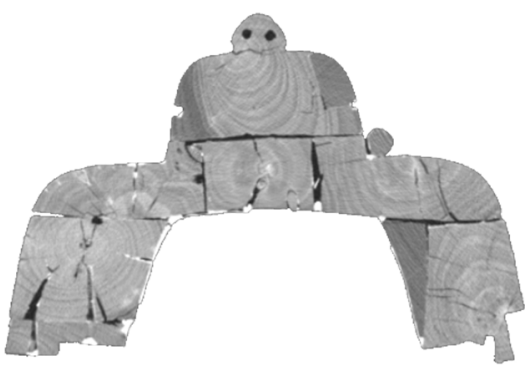

(b)

Figure 2. Three-dimensional exploration of the Pef's coffin interiors with X-Rays: (a) the computer tomography (CT) scanning of the coffin at the Galeazzi Hospital in Milan (Italy); (b) the section of the wooden coffin that allows to see the inner structure.

In particular, the CT investigation of Pef's mummy allowed us to identify both the main characteristics of the subject, who was 40 year old male, with physiognomic characteristics typical of African subjects and some peculiar characteristics related to his state of health, such as an impacted tooth, which is visible in Figure 9.

\subsection{Interactive Application}

The VR platform chosen for the museum application was Unity3D. Several reasons guided this choice: (i) its relatively widespread diffusion in the field of virtual museums; (ii) the powerful development system completely free of charge; (iii) the possibility to create small applications with no obligation to pay the company the royalties (something not sustainable in a small public museum); and last but not least, (iv) the existence of vibrant community-sharing approaches and ideas about specific technical solutions.

The pre-existing models, complemented by the new ones, were properly adapted in order to be usable in a Unity3D application. In particular, the best tradeoff was pursued between high resolution (for optimal visualization of the object's details) and good navigability in the virtual reality environment. When forced to reduce the mesh polygon density to prevent overloading the VR environment, we tried to maintain the details, at least visually, with a high-resolution texture, optimizing colors, shadows and contrasts. 
Early experiments with older models digitized within the 3D-ICONS initiative showed how high-resolution 3D content was suitable for immersive interaction with headsets and gesture tracking [49]. However, for various logistic and budgetary reasons described in Section 3, the museum pressured us to reduce the visualization and interaction technology, and to give stronger emphasis to creating rich visual content and an engaging narrative supported by reliable historical references.

Considering that the physical artifacts narrated in the application were on display at just a few meters from the digital installation, the museum and our team agreed to trade the "wow effect" of a stereoscopic view with a monoscopic visualization of the 3D content. We also had to forego modern gesture-tracking interaction in favor of a more straightforward touch screen. The latter choice was also made in consideration of the age of a good percentage of the public, who would have been uncomfortable with gesture tracking, if not wholly unable to deal with it.

The focus was then on the narrative structure of the virtual application, that was developed keeping in mind the "interplay between Form, Story, and History" theorized nearly 20 years ago by Maria Roussou [50], through an iterative and interdisciplinary process [51] with the participation of experts in digitalization, computer graphics, virtual reality, museum curators, Egyptologists, and anthropologists.

Such an initial discussion led to a narrative map [52], whose aim was to focus on the key points needed to explain the funeral ritual of ancient Egypt. The map has a tree structure with three main ramifications, corresponding to the fundamental requirements for obtaining eternal life:

1. Preserve the intact body through mummification, coffin, sarcophagus, and an appropriate tomb;

2. Feed the defunct with real or represented food;

3. Face ordeals with the help of rituals, amulets and coffin decorations as described in the papyrus of the "Book of the Dead" that was part of the burial goods.

As explained above, a proper subset of the whole museum collection was selected. A first mapping identifying all the semantic connections was achieved in a first release of the app, which supported the navigation that was both guided (following a predefined path) and free (with the user choosing the movement from one object to another).

The four canopic jars represent an excellent example of the connection between various elements of the funerary ritual. Each jar was reserved for safekeeping specific organs of the deceased: stomach, intestines, lungs, and liver, all of which, it was believed, would be needed in the afterlife. The corresponding lids depict four sons of the god Horus, as guardians of the organs: Duamutef, with a jackal's head, to protect the stomach; Kebehsenef, with a hawk's head, to protect the intestines, Hapy, with a baboon's head to protect lungs; Hamset, with a human's head, to protect the liver.

In addition to their presence in the collection as physical items, the canopic jars also appeared in many representations of the mummification scenes that decorated the coffin, or in some vignettes drawn in the Book of the Dead (Figure 3).

With reference to the extended graph of connection originated from the first mapping (Figure 4), the interim implementation led to two important issues potentially critical from the museum's point of view. First, the average time that each visitor might dedicate to the interaction could easily have exceeded a reasonable threshold because of the sheer number of connections between the objects, and this fact would have been a negative factor for the general public. Secondly, a rich hyperlinked structure with multiple media suitable for navigation, such as the 3D models, raised the possibility that users with little digital skill might get lost within the application, losing the sense of narration that was a key component of the desired outcome. 


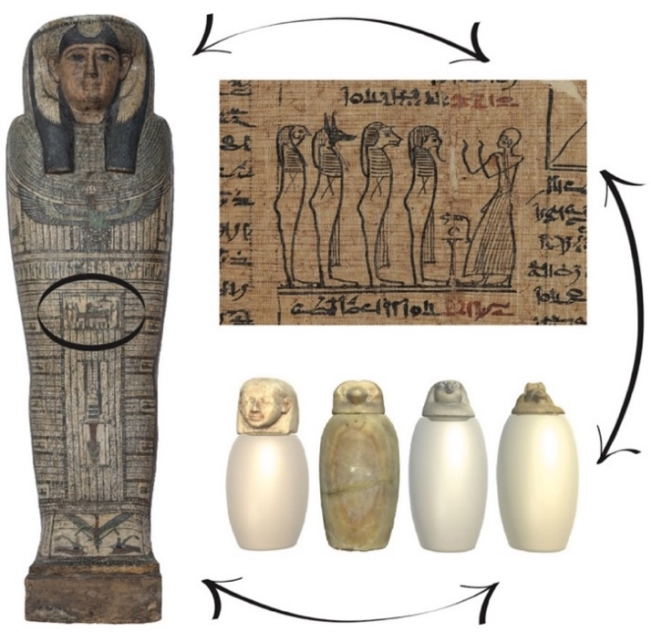

Figure 3. The image shows the different elements of the museum collection, selected for the digital application, which are mutually connected: the physical canopic jars and their representation in the vignette 124 of the Hornefer papyrus and on the decoration of a Tes coffin.

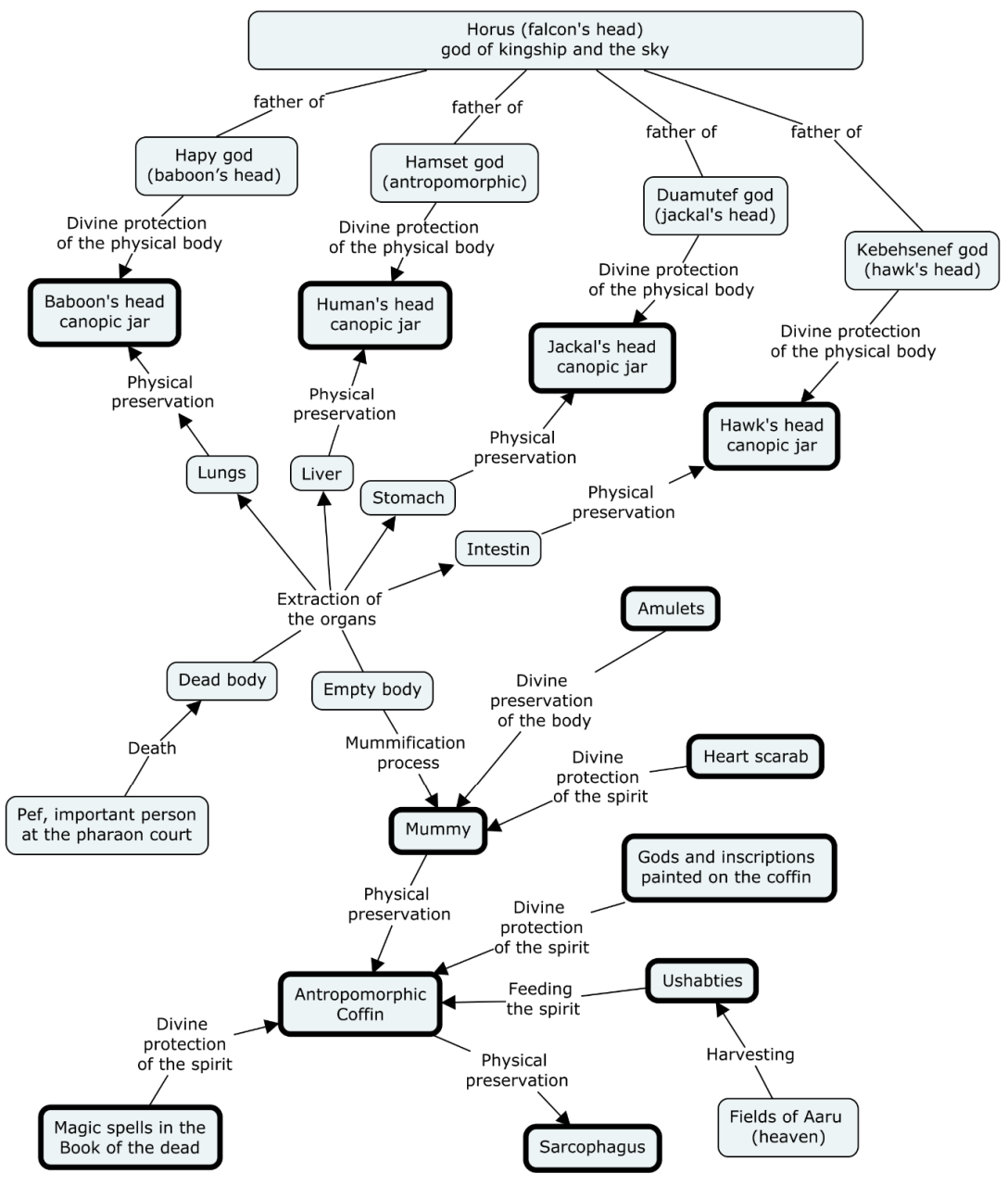

Figure 4. The graph connecting some of the actual objects in the exhibit (thick border), intangible heritage or no-longer extant objects (thin border) and the intangible heritage relations (connecting clauses). The Book of the Dead and the decorations over the coffin are linked with all the other items, but the connections are not represented for the sake of graphical clarity. 
To overcome these problems, the navigation mapping was re-designed, synthetizing the correct understanding provided by curators. In addition, an appropriate communication strategy was developed to ensure the correct use of the digital media as, for example, dedicated graphic icons, specific colors associated with the different themes and simplified interactions with 3D content.

Hence, the application has been divided into four levels, with an increasing depth of analysis and interaction modalities (Figure 5).

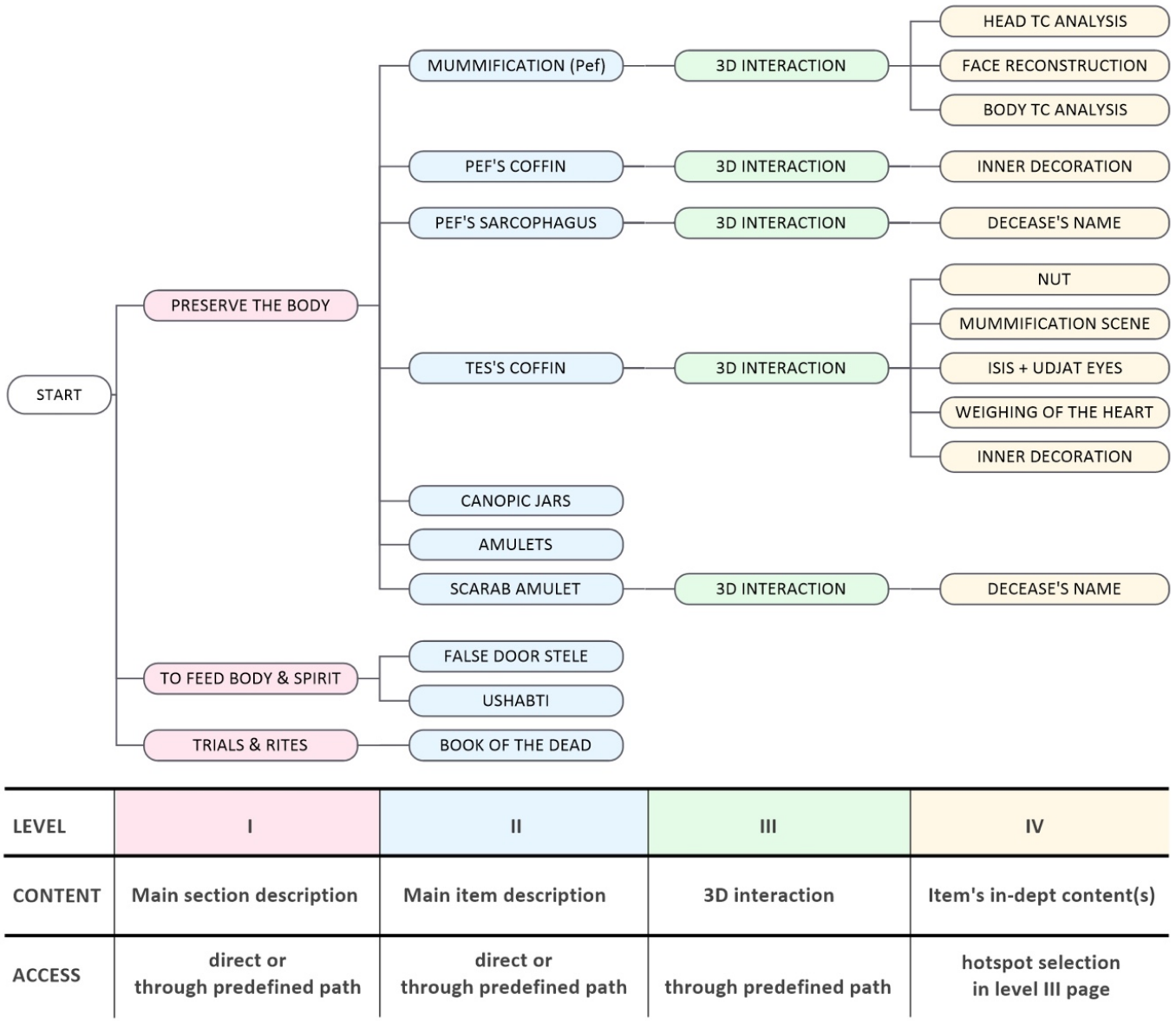

Figure 5. Diagram of the interactive digital application

Level I provides a description of the main sections. The content of this level includes a textual description of the section, with an interactive glossary of the uncommon or specialized words, and a representative image of the section. This level does not include interactive visualization items and is accessible both via direct icon selection and through the predefined path.

Level II describes the main items in the collection. Besides a textual description, the user in this level can have three different kinds of interaction according to the importance that the items have within the collection, the representation media and their frequency within the collection. Meaningful items such as the mummy or coffins, which include supplementary details available in Level III, are represented by a static representation (no interaction). Smaller items, such as ushabtis or canopic jars, with no supplementary information, are show by means of a small animation, which allows the user to have a quick overview of them (low interaction). When the items, instead, include multiple digital representations, they are arranged into an image-scroll panel, allowing the user to make an association between the items and their specific location into a proposed map (full interaction). This approach has been used, for example, to show a selection of the hundreds of mummy amulets (Figure 6) and a selection of the hundreds of vignettes from the Book of the Dead. As with Level I, it is accessible via direct icon selection or through the predefined navigation path. 
Level III is the one with the most advanced degree of interaction, and it allows the user to discover more details about the proposed object. Here, an animation of the 3D model shows the most significant perspectives of the item. At the end of the animation, the user can interact with the item through these three elements:

- Hotspots to allow the user to enable in-depth pages (Level IV) associated to their location on the 3D model. The visualization of these hotspots can be switched through a button;

- A slider to navigate all the perspectives of the introductory animation;

- A magnifying glass to appreciate the details of the 3D model.

In general, these tools have been developed with the aim of manipulating the 3D model and displaying the most interesting areas by reducing the chance that users can get lost in the navigation within the three-dimensional space. Level III is accessible only through the predefined path, from the corresponding page on Level II.

Level IV consists of the in-depth pages that provide details about the single object. Each page concentrates on a specific topic, and depending on the content, different media are used to increase its understanding. For example, the videos of the CT accompanied by an explanatory text were used to describe the information processed by anthropologists based on the medical analyses performed on the mummy. In contrast, an image with the simplified reproduction of the signs, their transliteration and the translation into contemporary language, are used to explain the meaning of some hieroglyphics. Level IV is accessible by selecting the hotspot connected to the 3D model on the Level III page.

Therefore, depending on the desired level of deepening and the available time, the user can choose their own navigation approach.

An estimation of the time required by the different navigation modalities is:

- $\quad$ Three minutes for a synthetic overview of the whole material (Level I);

- Twenty to twenty-five minutes for a complete navigation.

The display area layout (Figure 7), designed for a 32-inch touchscreen monitor with a 16:9 horizontal aspect ratio, has two main portions: a central area dedicated to the experience of the content (texts, 3D models, images, videos, etc.) with the same extension in all levels; a frame area, on the sides and at the bottom of the screen, that contain all the interactive elements for page navigation and content use, distinguished according to the level.

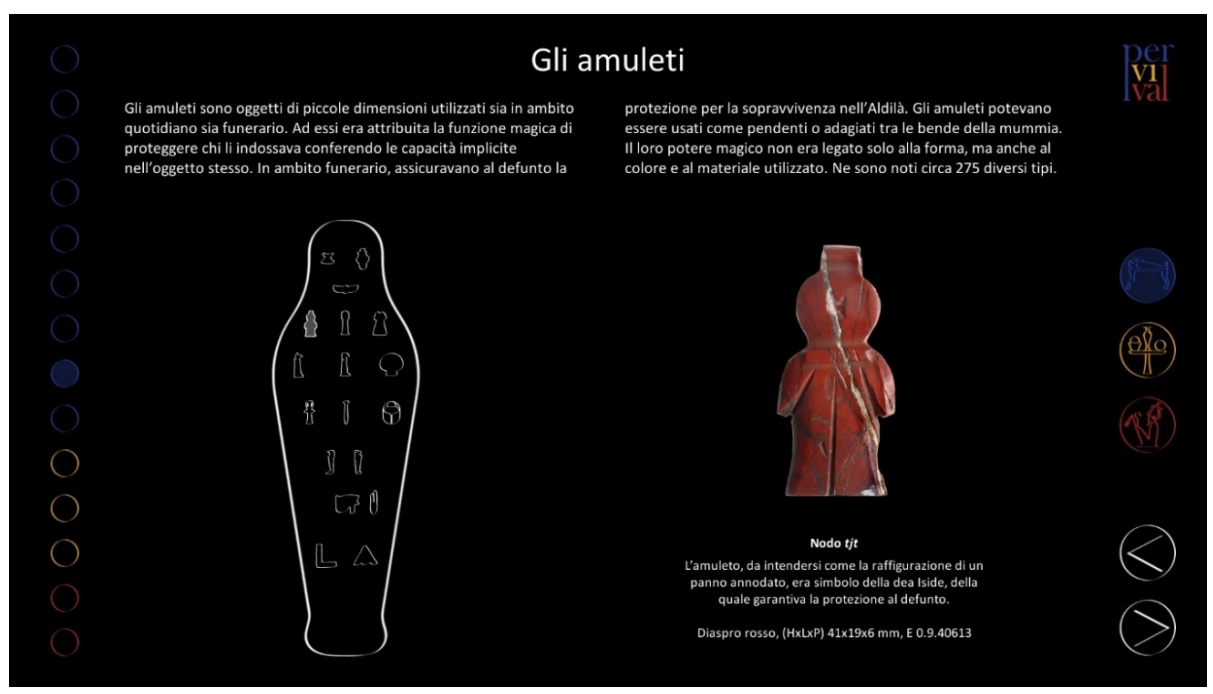

Figure 6. Digital app interface showing an example of the navigation Level II page with the main description of the single items. In the amulets page, the detailed information about the individual amulets is visible by scrolling through them on the right or by selecting the icon on the mummy map. 
Most of the pages (Level I, II, III) share the same setting: on the left side, are the buttons to directly access the Level II pages; on the right side, are the buttons to access the home page, the main section pages of Level I and to move forward or backward in the predefined navigation path.

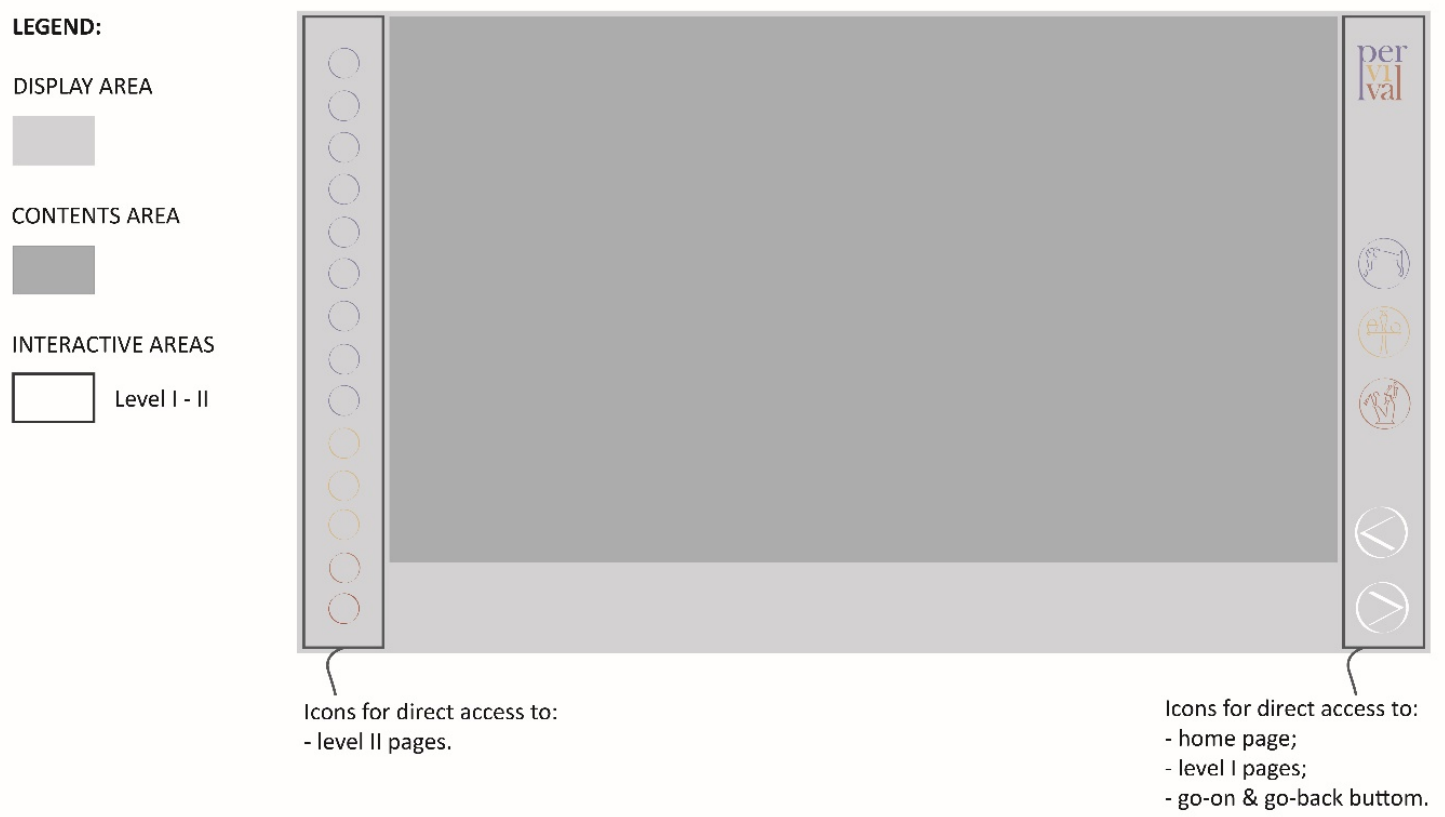

Figure 7. The layout of the digital application, referred to as Level I, II and III.

In Level III (Figure 8), a bar is added to the base of the display, with tools for direct interaction with the model: the on/off switch, represented as a little lamp, that activates various hotspots over the 3D model for in-depth explorations of specific areas of the object; the slider at the bottom of the figure that allows replaying the 3D model rotation already proposed automatically by the app during the browsing; the "+" button that activates the magnifying glass manageable by the user, for zooming locally over the 3D model.

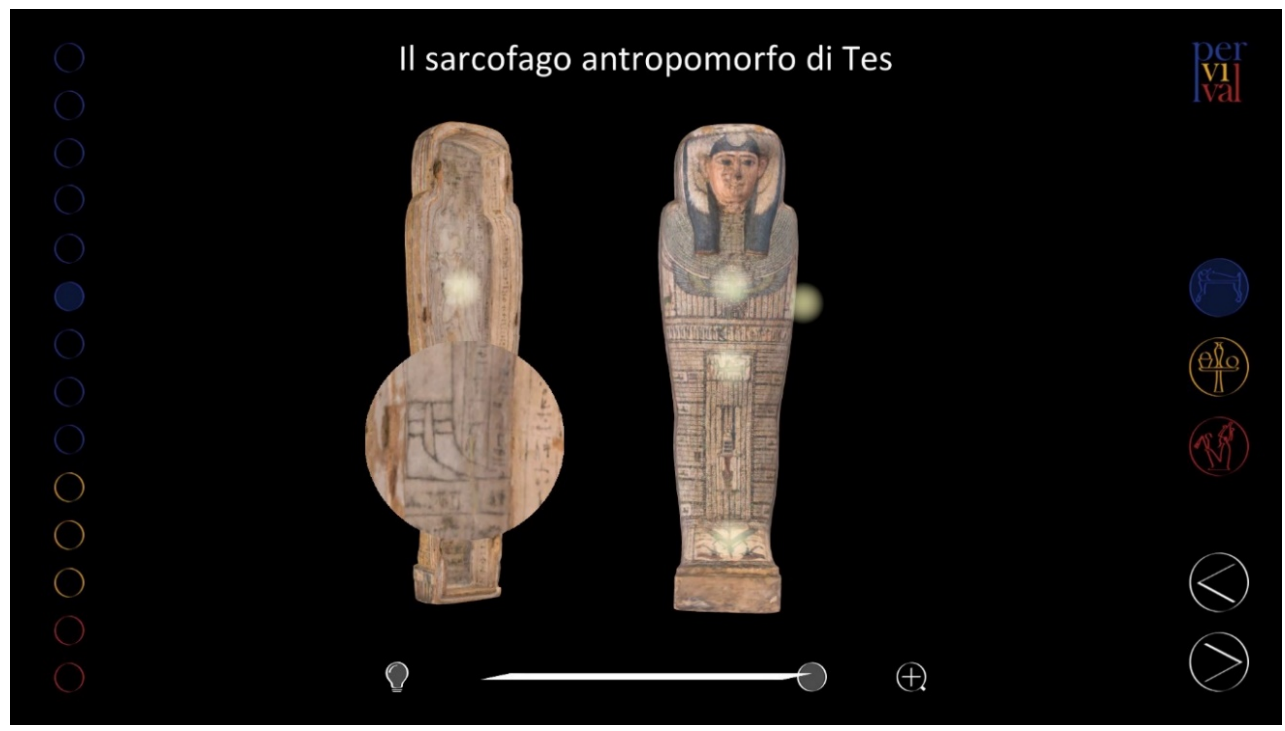

Figure 8. Digital app interface, navigation Level III page: single item automatic rotation and access to the interactive elements: hotspot selector, slider to replay the rotation, magnifying glass. 
The Level IV pages are those with simpler layout. In addition to the item's insight content, they have only the closing button of the page itself in the lower right corner (Figure 9).

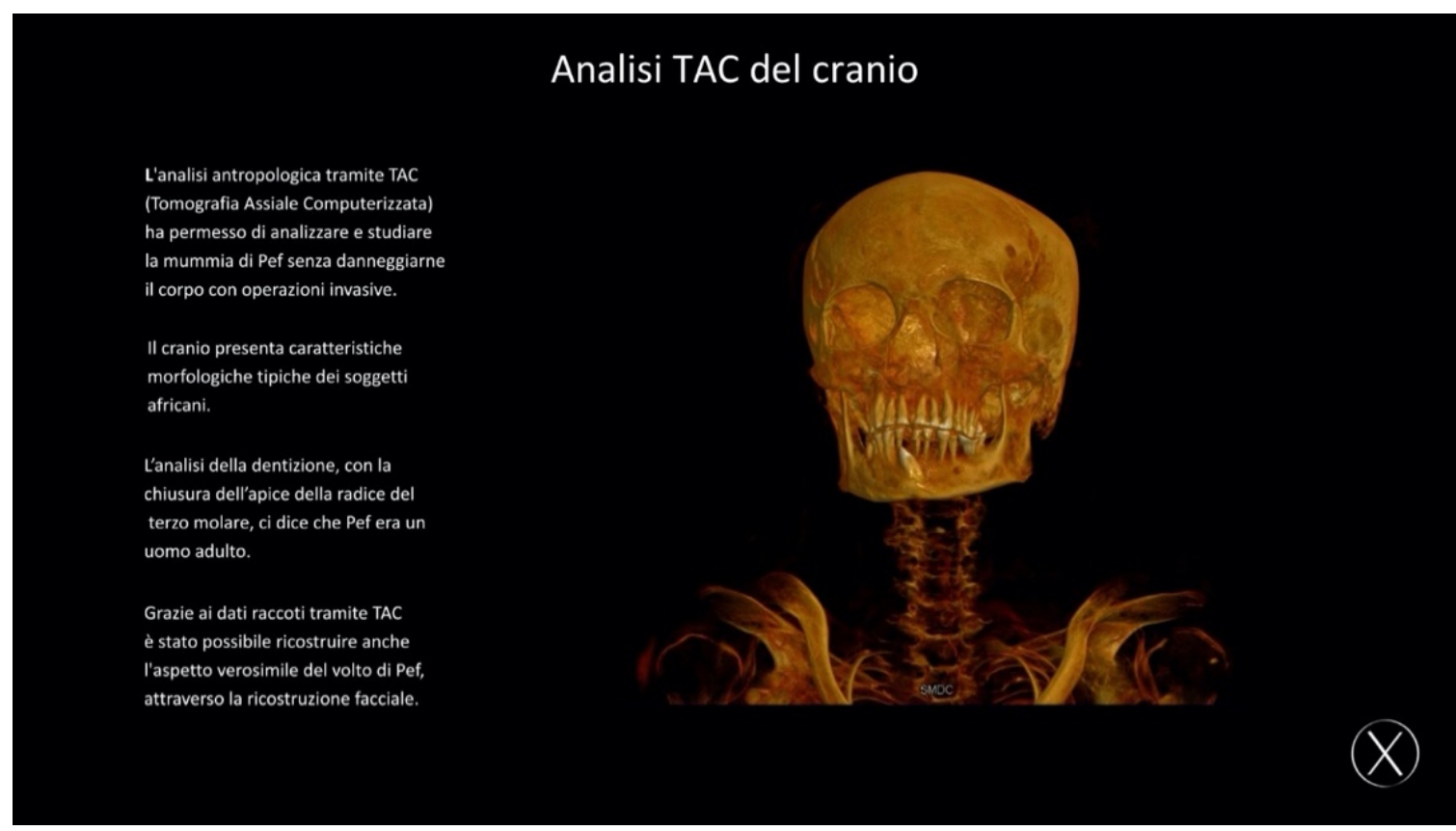

Figure 9. Images of the digital app interface at navigation Level IV, showing an item's in-depth contents: CT scan video of a mummy's head with textual explanation of the information that can be drawn from medical analyses, as for example the evident impacted tooth.

In designing the layout, the system usability by museum visitors with limited access to the upper parts of the screen was also considered [31]. For example, the buttons for navigating the predefined path and the closing button of the hotspot pages have been deliberately placed in the lower right corner of the display area. In this way, they are closer to the floor, and both a child or an adult in a wheelchair can easily access them. Being close to each other, the interaction involves lower fatigue.

Furthermore, the icons of the elements with which the user can interact have been sized large enough to be easily selectable, occupying an area between 20 and $40 \mathrm{~mm}$ on the display.

\section{Discussion and Conclusions}

In recent years, we have witnessed the multiplication of technological applications in the museum field that implement advanced solutions based on spectacular technologies to involve visitors such as augmented reality [17], storytelling [16,27,37] and concepts such as participation [53] and personalized visit [28], which are certainly transforming museum curation and the way museums relate to visitors.

In the research described in this article, we analyzed the design strategies for approaching an interactive application illustrating a "complex museum collection" (CMC), here defined as a collection of cultural heritage pieces linked each other through intangible cultural heritage (ICH) elements, not exhibited in the museum for their intrinsic immaterial nature. In order to clarify the concepts, this paper shows a practical example based on a collection of grave goods from ancient Egypt. The result is an effective synthesis of an intricate network of semantic meanings in a friendly touch-screen based interactive application suitable for a broad range of end-users, including people with little or no technological skill. It makes "readable" a collection of amulets, mummies, coffins and the related inscriptions through the explication of their hidden meanings associated with the articulated funerary rituals of the ancient Egypt.

The implementation was developed despite the technological trend mentioned above: instead, it was based on a simple interactive kiosk. Two reasons explained this choice: the need to keep the 
project and its maintenance low to take into account the economic constraints on Italian museums; and, more positively, the conscious design choice connected to the type of collection considered.

Any collection of a lower level of complexity can be experienced by a visitor more engagingly by walking in the corridors of the museum, and finding targeted explanations from time to time. It is the same criterion inspiring audio guides or the most modern augmented reality systems, which integrate the museum tour with location-dependent pieces of information aimed at explaining what is being observed at that moment.

In the case of $\mathrm{CMCs}$, what emerged from the interaction among the members of the interdisciplinary team is the insight that a place of preparation for the visit would be more useful than simply walking around physical objects and reading the related labels. In this way, a series of concepts, characters, divinities, and elements of ICH such as beliefs or knowledge related to the realization of specific processes (e.g., mummification) can be made explicit before the actual visit.

In such a context, the ICH component could itself be the object of representation using a "cultural experience room" similar to that proposed by Gottlieb in 2014 [54]. However, rather than providing a display for the various ICH components of the CMC collection, our research aimed at determining how to make the visitor aware of the stage on which the ritual action was performed with the objects on display, creating an expectation that is satisfied during the visit itself.

In a case like this, making the connections between the exhibited pieces explicit, hidden behind the articulated ICH elements, intertwined among the items in the collection, is more effective than other possible forms of engagement such as storytelling or personalization that, admittedly, work well in different contexts [55,56].

Nevertheless, the idea of a preparatory phase based on digital aids to prepare the visit of a cultural asset is not new; it works both to create the right cultural setup and to maximize the visitor's perceptive receptivity. As stated by Daniel Goleman in a recent interview, "One interesting question is, how, as someone enters a museum or a gallery, can we change their mental state so that they're in this focused, calm, and receptive mindset? This means leaving behind the state of mind that people are ordinarily in while they are walking down the street, or in their offices, or in their homes. In other words, we tend to live in a universe of mental distraction, lack of focus, where we pay attention to whatever is most dominant or most pressing-or actually what is most emotionally upsetting. It turns out that people spend most of their free time with their minds wandering to problems they are having in relationships. You want people to leave that mind behind as they enter the exhibit (... ) That's what virtual reality does, it creates a world that you want them to perceive." [57].

The methodology tested in this article explores this concept of virtual preparation for the physical visit. It lets the visitor better understand the collection they are about to visit, also entering in an emotional relationship with the pieces on display. The thesis is that with a CMC, an interactive starting area for a visit can be more effective than other MR contributions, given the highly symbolic role of the exhibited objects as well as their involvement in ritual actions far from our culture and therefore cryptic for a modern visitor.

A solution of this kind is also easily scalable. The example in this article presents an implementation with a touchscreen. Nonetheless, depending on the budget and available spaces, we could think of moving from an application of this kind to one based on multiple touchscreens, or to a dedicated room with wearable viewers that show the same contents in VR and with a gesture-based interface, without changing, in fact, the sense of the project.

As a future development of this project, it will be interesting to explore the number and quality of the interactions. For this purpose, the app is able to log anonymously all the navigation data associated with the different users. By statistically analyzing such data log with deep-learning tools, it should be possible to discover branches of the navigation less visited than others, misleading parts (if any), and other potential issues. In addition to traditional tests based on statistics and possible qualitative analysis on selected groups of end-users, this could provide an innovative methodological 
path for assessing interaction effectiveness, also providing directions for the continuous improvement of the application.

Author Contributions: Conceptualization, L.L.M.; G.C. and G.G.; methodology, G.G.; software, G.C.; visualization, L.L.M.; resources, L.L.M. and G.G.; data curation, L.L.M.; writing-original draft preparation, L.L.M., G.C. and G.G.; writing - review and editing, G.G.; project administration, L.L.M.; funding acquisition, G.G. All authors have read and agreed to the published version of the manuscript.

Funding: This research and the APC was funded by Fondazione CARIPLO, grant number 2017-1766.

Acknowledgments: The authors would like to acknowledge the collaborators, the Egyptologist Alice Sbriglio for the consultancy on Egyptian funerary rituals and the texts redaction of the virtual app and Sara Gonizzi for some 3D survey works. Furthermore, they would like to thank the following institutions and their staff without whose collaboration PERVIVAL would not have been possible: the Civic Archaeological Museum of Milan, for granting access to the Egyptian material, its Director Anna Provenzali, the Egyptologists Sara Loreto and Sabrina Ceruti for information provided about the Egyptian collection; the IRCCS (Istituto Ortopedico Galeazzi di Milano) for the CT survey of the mummy and the coffin of Peftjauauiaset, and its staff members Grazia Pozzi, Luca Maria Sconfienza and Ernesto Pregliasco.; the LABANOF of the University of Milan for the interpretation of the clinical data acquired by $\mathrm{CT}$ and for the 3D physical reconstruction of Peftjauauiaset's face, with special thanks to Davide Porta, Cristina Cattaneo, Debora Mazzarelli and Pasquale Poppa.

Conflicts of Interest: The authors declare no conflict of interest.

\section{References}

1. Museum Associations. Museums of the World 2019; De Gruyter Saur: Berlin, Germany, 2019; ISBN 978-311-063555-3.

2. ICOM Museum Definition. Available online: https://icom.museum/en/resources/standards-guidelines/ museum-definition/ (accessed on 25 May 2020).

3. Jenkins, H. Convergence Culture: Where Old and New Media Collide; New York University Press: New York, NY, USA, 2016; ISBN 978-0-8147-4281-5.

4. Bedford, L. Storytelling: The Real Work of Museums. CURA Curator Mus. J. 2001, 44, 27-34. [CrossRef]

5. Parry, R. Museums in a Digital Age; Routledge: London, UK, 2010; ISBN 0-415-40261-1.

6. Geismar, H. (Ed.) Museum Object Lessons for the Digital Age; UCL Press: London, UK, 2018; ISBN 978-178735-281-0

7. Milgram, P.; Takemura, H.; Utsumi, A.; Kishino, F. Augmented reality: A class of displays on the reality-virtuality continuum. SPIE 1995, 2351, 282-292.

8. Bekele, M.K.; Champion, E. A comparison of immersive realities and interaction methods: Cultural learning in virtual heritage. Front. Robot. AI 2019, 6. [CrossRef]

9. Petrelli, D. Making virtual reconstructions part of the visit: An exploratory study. Digit. Appl. Archaeol. Cult. Herit. 2019, 15, e00123. [CrossRef]

10. Kiourt, C.; Koutsoudis, A.; Pavlidis, G. DynaMus: A fully dynamic 3D virtual museum framework. J. Cult. Herit. 2016, 22, 984-991. [CrossRef]

11. Hornecker, E.; Ciolfi, L. Human-Computer Interactions in Museums: Synthesis Lectures on Human-Centered Informatics; Morgan \& Claypool: San Rafael, CA, USA, 2019; ISBN 978-1-68173-513-9.

12. Hoffman, M.; Law, C.; Psihogios, J.; Vanderheiden, G.; Ziebarth, B. Development of accessible kiosk user interface solutions for the public sector: A panel summary. In Proceedings of the Human Factors and Ergonomics Society Annual Meeting, Minneapolis, MN, USA, 8-12 October 2001; pp. 230-234.

13. Burmistrov, I. Touchscreen Kiosks in Museums. Available online: https://doi.org/10.13140/rg.2.1.4521.0087 (accessed on 19 June 2020).

14. Carrozzino, M.; Bergamasco, M. Beyond virtual museums: Experiencing immersive virtual reality in real museums. J. Cult. Herit. 2010, 11, 452-458. [CrossRef]

15. Marín-Morales, J.; Higuera-Trujillo, J.L.; De-Juan-Ripoll, C.; Llinares, C.; Guixeres, J.; Iñarra, S.; Alcañiz, M. Navigation comparison between a real and a virtual museum: Time-dependent differences using a head mounted display. Interact. Comput. 2019, 31, 208-220. [CrossRef]

16. Nack, F.; Waern, A. Mobile digital interactive storytelling-A winding path. New Rev. Hypermedia Multimed. 2012, 18, 3-9. [CrossRef] 
17. Serravalle, F.; Ferraris, A.; Vrontis, D.; Thrassou, A.; Christofi, M. Augmented reality in the tourism industry: A multi-stakeholder analysis of museums. Tour. Manag. Perspect. 2019, 32, 100549. [CrossRef]

18. Khan, D.; Ullah, S.; Yan, D.; Rabbi, I.; Richard, P.; Hoang, T.; Billinghurst, M.; Zhang, X. Robust tracking through the design of high quality fiducial markers: An optimization tool for artoolkit. IEEE Access 2018, 6, 22421-22433. [CrossRef]

19. vGIS Team 2020 iPad Pro: Does the LiDAR Sensor Improve Spatial Tracking? Available online: https: //www.vgis.io/2020/04/23/2020-ipad-pro-does-the-lidar-sensor-improve-spatial-tracking/ (accessed on 30 May 2020).

20. Sullivan, M. The Next iPhone will Get a 'World Facing' 3D Camera. Available online: https://www. fastcompany.com/90474966/the-next-iphone-will-get-a-world-facing-3d-camera (accessed on 30 May 2020).

21. Kenderdine, S.; Chan, L.K.Y.; Shaw, J. Pure Land: Futures for embodied museography. J. Comput. Cult. Herit. 2014, 7, 1-15. [CrossRef]

22. Tsichritzis, D.; Gibbs, S. Virtual museums and virtual realities. In Proceedings of the International Conference on Hypermedia and Interactivity in Museums, Pittsburgh, PA, USA, 14-16 October 1991; pp. 17-25.

23. Roussou, M. High-End Interactive Media in the Museum. In ACM SIGGRAPH 99 Conference Abstracts and Applications, Proceedings of the SIGGRAPH99: 26th International Conference on Computer Graphics and Interactive Techniques, Los Angeles, CA, USA, 8-13 August 1999; Association for Computing Machinery: New York, NY, USA, 1999; pp. 59-62.

24. Parent, A. A Virtual Environment Task-Analysis Tool for the Creation of Virtual Art Exhibits. Presence Teleoperators Virtual Environ. 1999, 8, 355-365. [CrossRef]

25. Network of European Museum Organization (NEMO) EU funding and Museums. Available online: https://www.ne-mo.org/fileadmin/Dateien/public/NEMO_documents/NEMO_EU_funding_and_ Museums_report.pdf (accessed on 27 May 2020).

26. White, M.; Chmielewski, J.; Stawniak, M.; Wiza, W.; Patel, M.; Stevenson, J.; Manley, J.; Giorgini, F.; Sayd, P.; Gaspard, F.; et al. ARCO—An architecture for digitization, management and presentation of virtual exhibitions. In Proceedings Computer Graphics International; IEEE: Crete, Greece, 2004; pp. 622-625.

27. Katifori, A.; Karvounis, M.; Kourtis, V.; Kyriakidi, M.; Roussou, M.; Tsangaris, M.; Vayanou, M.; Ioannidis, Y.; Balet, O.; Prados, T.; et al. CHESS: Personalized Storytelling Experiences in Museums. In Interactive Storytelling; Mitchell, A., Fernández-Vara, C., Thue, D., Eds.; Springer International Publishing: Cham, Switzerland, 2014; pp. 232-235.

28. Roussou, M.; Katifori, A. Flow, Staging, Wayfinding, Personalization: Evaluating User Experience with Mobile Museum Narratives. Multimodal Technol. Interact. 2018, 2, 32. [CrossRef]

29. Marshall, M.T.; Dulake, N.; Ciolfi, L.; Duranti, D.; Kockelkorn, H.; Petrelli, D. Using Tangible Smart Replicas as Controls for an Interactive Museum Exhibition. In TEI '16: Tenth International Conference on Tangible, Embedded, and Embodied Interaction; Association for Computing Machinery: New York, NY, USA, 2016; pp. 159-167.

30. The United Nations Educational, Scientific and Cultural Organization. Convention for the Safeguarding of the Intangible Cultural Heritage; UNESCO: Paris, France, 2003.

31. Kirshenblatt-Gimblett, B. Intangible Heritage as Metacultural Production. Mus. Int. 2004, 56, 52-65. [CrossRef]

32. Carboni, N.; de Luca, L. Towards a conceptual foundation for documenting tangible and intangible elements of a cultural object. Digit. Appl. Archaeol. Cult. Herit. 2016, 3, 108-116. [CrossRef]

33. Foucault, M. The Order of Things; Routledge: London, UK, 2018; ISBN 978-1-315-66030-1.

34. Macdonald, S. Collecting practices. In A Companion to Museum Studies; Macdonald, S., Ed.; Wiley-Blackwell: Hoboken, NJ, USA, 2007; ISBN 978-1-4051-0839-3.

35. Bennett, T. Pasts Beyond Memory: Evolution, Museums, Colonialism; Routledge: London, UK, 2004; ISBN 0-41524746-2.

36. Knell, S.J. Museums and the Future of Collecting; Routledge: London, UK, 2016; ISBN 978-1-351-91643-1.

37. Katifori, A.; Karvounis, M.; Kourtis, V.; Perry, S.; Roussou, M.; Ioanidis, Y. Applying Interactive Storytelling in Cultural Heritage: Opportunities, Challenges and Lessons Learned. In Interactive Storytelling; Rouse, R., Koenitz, H., Haahr, M., Eds.; Springer International Publishing: Cham, Switzerland, 2018; pp. 603-612.

38. Redford, D.B. The Oxford Encyclopedia of Ancient Egypt; Oxford University Press: Oxford, UK, 2001; ISBN 0-19-513821-X. 
39. Foy, S.; Lowry, K.B. Book of the Dead: Becoming God in Ancient Egypt; Oriental Institute of the University of Chicago: Chicago, IL, USA, 2017; ISBN 978-1-61491-038-1.

40. Pinch, G. Egyptian Mythology: A Guide to the Gods, Goddesses, and Traditions of Ancient Egypt; Oxford University Press: Oxford, UK, 2004; ISBN 0-19-517024-5.

41. Andrews, C. Amulets of Ancient Egypt; University of Texas Press: Austin, TX, USA, 1994; ISBN 0-292-70464-X.

42. Stewart, H.M. Egyptian Shabtis; Shire: Lexington, MA, USA, 1997; ISBN 0-7478-0301-3.

43. Lise, G. Museo Archeologico: Raccolta Egizia; Electa: Milan, Italy, 1979.

44. Miatello, L. Examining Texts and Decoration of Peftjauauiaset's Coffins in Milan. Égypte Nil. Médit. ENIM 2018, 11, 41-133.

45. Cignoni, P.; Callieri, M.; Corsini, M.; Dellepiane, M.; Ganovelli, F.; Ranzuglia, G. MeshLab: An open-source mesh processing tool. In Proceedings of the 6th Eurographics Italian Chapter Conference, Salerno, Italy, 2-4 July 2008.

46. Guidi, G.; Gonizzi Barsanti, S.; Micoli, L.L.; Russo, M. Massive 3D Digitization of Museum Contents. In Built Heritage: Monitoring Conservation Management; Toniolo, L., Boriani, M., Guidi, G., Eds.; Springer International Publishing: Cham, Switzerland, 2015; pp. 335-346. ISBN 978-3-319-08533-3.

47. Lucarelli, R. Images of Eternity in 3D. The visualization of ancient Egyptian coffins through photogrammetry. In Altertumswissenschaften in A Digital Age: Egyptology, Papyrology and beyond; Berti, M., Naether, F., Eds.; University of Leipzig: Leipzig, Germany, 2015; pp. 24.1-24.3.

48. Jackowski, C.; Bolliger, S.; Thali, M.J. Scenes from the past-Common and unexpected findings in mummies from ancient Egypt and South America as revealed by CT. Radiographics 2008, 28, 1477-1492. [CrossRef] [PubMed]

49. Gonizzi Barsanti, S.; Caruso, G.; Guidi, G. Virtual navigation in the ancient Egyptian funerary rituals. In Proceedings of the 2016 22nd International Conference on Virtual System Multimedia (VSMM), Otranto, Italy, 17 October 2016; pp. 1-6.

50. Roussou, M. The Interplay between Form, Story, and History: The Use of Narrative in Cultural and Educational Virtual Reality. In International Conference on Virtual Storytelling: Using Virtual Reality Technologies for Storytelling; Springer: Berlin, Germany, 2001; pp. 181-190.

51. Mason, M. Prototyping practices supporting interdisciplinary collaboration in digital media design for museums. Mus. Manag. Curatorship 2015, 30, 394-426. [CrossRef]

52. Landa, J.A.G. In Hindsight: Complexity, Contingency, and Narrative Mapping. In Narrative Complexity: Cognition, Embodiment, Evolution; Grishakova, M., Poulaki, M., Eds.; University of Nebraska Press: Lincoln, NE, USA, 2019; pp. 414-436. ISBN 978-0-8032-9686-2.

53. Simon, N. The Partecipatory Museum; Museum 2.0: Santa Cruz, CA, USA, 2010; ISBN 978-0-615-34650-2.

54. Ng, B.C.; Cavallaro, F.; Cl, L.; Gottlieb, H. A Cultural Experience Room: An Interdisciplinary and Inclusive Approach to Visualising Intangible Heritage. In Engaging Spaces: Interpretation, Design and Digital Strategies; Muzeum of King Jan III's Palace at Wilanow: Poznan, Poland, 2014; pp. 306-319.

55. Muñoz, A.; Martí, A. New Storytelling for Archaeological Museums Based on Augmented Reality Glasses. In Communicating the Past in the Digital Age; Hageneuer, S., Ed.; Ubiquity Press: London, UK, 2020; pp. 85-100. ISBN 978-1-911529-84-2.

56. Harrington, M.C.R.; Tatzgern, M.; Langer, T.; Wenzel, J.W. Augmented Reality Brings the Real World into Natural History Dioramas with Data Visualizations and Bioacoustics at the Carnegie Museum of Natural History. Curator Mus. J. 2019, 62, 177-193. [CrossRef]

57. Falvo, P.G.; Manera, G.V.; Zoss, J. Conversation with Daniel Goleman about the relationship between the person viewing art and the art itself. Stud. Digit. Herit. 2018, 2. [CrossRef]

(C) 2020 by the authors. Licensee MDPI, Basel, Switzerland. This article is an open access article distributed under the terms and conditions of the Creative Commons Attribution (CC BY) license (http://creativecommons.org/licenses/by/4.0/). 